\title{
MILLIMETER OBSERVATIONS OF G5.89-0.39
}

\author{
DAVID J. WILNER AND W. J. WELCH \\ Astronomy Dept., University of California, Berkeley, CA 94720 USA
}

J. R. FORSTER

Hat Creek Radio Observatory, Rt. 2, Box 500, Cassel, CA 96016 USA

YASUHIRO MURATA

Deep Space Communication Research, Institute of Space and Astronautical Science, 1-1, Yoshinodai 3-chome, Sagamihara, Kanagawa 229 Japan

ABSTRACT We present multi-line millimeter interferometer maps and single dish observations of the G5.89-0.39 ultracompact HII region. Radiative transfer modelling suggests an overall collapse.

\section{OBSERVATIONS}

The G5.89 ultracompact HII region is particularly well suited to detailed study because it is one of the closest $(\mathrm{d} \sim 3 \mathrm{kpc})$, most luminous $\left(\mathrm{L} \sim 3 \times 10^{5} \mathrm{~L}_{\odot}\right)$ and most morphologically simple of the known ultracompact HII regions (Wood and Churchwell 1989). Nine transitions in five molecular species, continuum emission, and a recombination line were mapped toward G5.89 with the Hat Creek interferometer $\left(\mathrm{HCO}^{+}{ }_{1-0} \mathrm{H}^{13} \mathrm{CN} 1-0\right.$, SO $\left.2_{2}-1_{1},{ }^{12} \mathrm{CO} 1-0\right)$ and the Nobeyama Millimeter Array ( $\left.\mathrm{H} 41 \alpha, \mathrm{CH}_{3} \mathrm{CN} 5-4, \mathrm{~K}=0,1,2,3,4\right)$. The highest spatial resolution was obtained with the NMA, where uniform weighting yielded a $2.7 \times 1.7$ synthesized beam (Figure I).

\section{G5.89 KINEMATICS}

Our ${ }^{12} \mathrm{CO} \mathrm{J}=1-0$ interferometer maps and $\mathrm{J}=2-1$ and $\mathrm{J}=3-2$ single dish spectra constrain the ${ }^{12} \mathrm{CO}$ excitation and confirm earlier estimates that the G5.89 bipolar outflow is the most powerful yet discovered (Harvey and Forveille 1988). The $\mathrm{HCO}^{+} 1-0$ spectrum toward the $\mathrm{HII}$ region shows two redshifted absorption features (Figure II). The strength of the redder feature does not change relative to the continuum with increasing beam size, indicating that there is no emission associated with this feature; this absorption is probably due to an unrelated foreground cloud. In contrast, there is emission associated with the lower velocity absorption system. Since this requires high densities $\left(\gtrsim 10^{5} \mathrm{~cm}^{-3}\right)$, this absorbing material is likely dynamically related to the G5.89 core. We have modelled the molecular envelope with the radiative transfer code described by Auer and Dickel (1988) and find that the detailed distributions of $\mathrm{HCO}^{+} \mathrm{J}=1-0$ and $\mathrm{J}=3-2$ emission and absorption are well matched by remnant infall. 


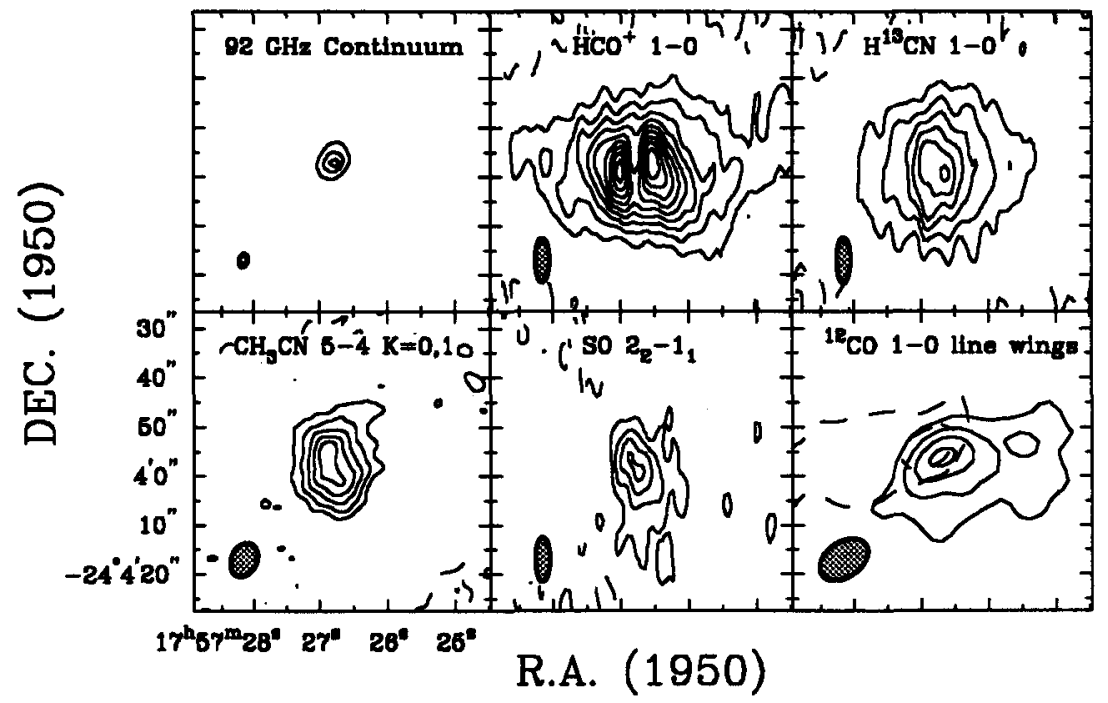

FIGURE I Integrated intensity maps toward G5.89. Continuum emission has been subtracted from the molecular maps. The contour interval and lowest contour are $1.5 \mathrm{~K}$ for $\mathrm{HCO}^{+}, \mathrm{H}^{13} \mathrm{CN}$ and $\mathrm{SO}, 0.5 \mathrm{~K}$ for $\mathrm{CH}_{3} \mathrm{CN}, 7 \mathrm{~K}$ and $35 \mathrm{~K}$ for the continuum, and $2.4 \mathrm{~K}$ for the ${ }^{12} \mathrm{CO}$ line wings $\left([30,60] \mathrm{km} \mathrm{s}^{-1}\right.$ dashed, $[-40,-10] \mathrm{km} \mathrm{s}^{-1}$ solid).
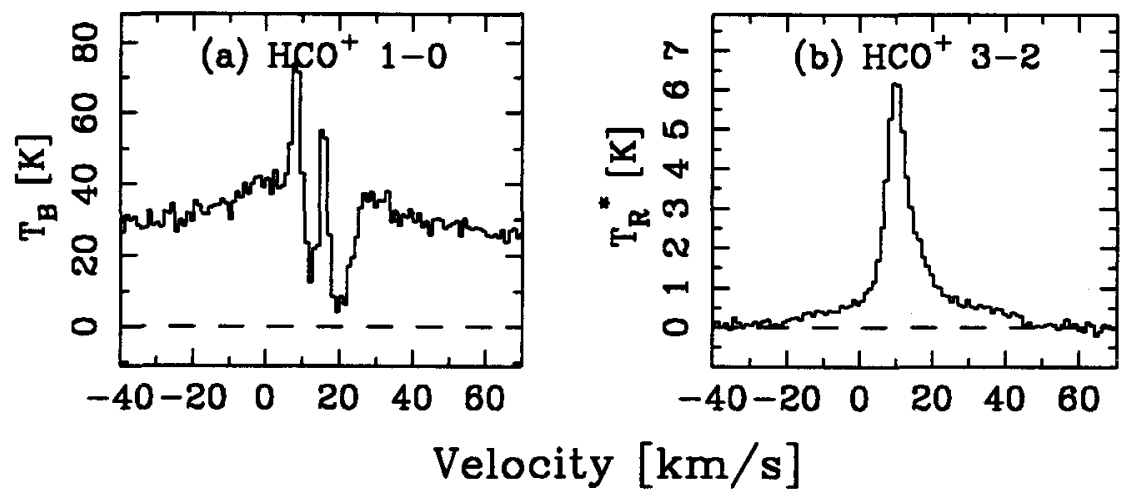

FIGURE II $\mathrm{HCO}^{+}$spectra toward G5.89. (a) $\mathrm{J}=1-0$ profile from the Hat Creek array; beam $8.77 \times 2 ! 8$. (b) J=3-2 profile from the NRAO $12 \mathrm{~m}$ telescope, continuum subtracted; beam 24 ".

\section{REFERENCES}

Auer, L.H., and Dickel, H.R. 1988, in Interstellar Matters, eds. J. Moran and P.T.P. Ho, New York: Gorden \& Breach, p. 165

Harvey, P., and Forveille, T. 1988, A\&A 197, L19

Wood, D.O.S., and Churchwell, E. 1989, ApJS 69, 831 